\title{
DISCUSSION
}

\section{Learning patterns and learner profiles in learning object design}

\author{
A reply to Andy Heath's response 'Exploiting \\ opportunities computers provide to deliver content \\ people can use in context'
}

Steve Green ${ }^{\mathrm{a} *}$, Ray Jones ${ }^{\mathrm{b}}$, Elaine Pearson ${ }^{\mathrm{a}}$ and Stavroula Gkatzidou $^{\mathrm{a}}$

${ }^{\mathrm{a}}$ University of Teesside, UK; ${ }^{\mathrm{b}}$ London Metropolitan University, UK

The questions that Andy Heath has posed are challenging and need more time for reflection than is possible here. The questions posed will inform the research as it develops further. However, in the interests of debate we would like to give our initial replies.

We agree in general with Andy Heath's assessment of the limitations of the approach we are adopting. We recognise that this approach uses a very limited response to AccessForAll principles: our Transformation Augmentation and Substitution Service (TASS) is localised, not global, and relies on a limited set of learning patterns matched against a small subset of the potentially infinite set of learner profiles. Our intention is certainly not to reproduce the considerable efforts of the IMS AccessForAll or Dublin Core Adaptability working groups, but to interpret their potential impact on the thinking of courseware designers, tutors and students.

Our ultimate aim is to broaden the service, but we need to begin with a limited set, working on known profiles, so that the more general requirements can be determined. Also we are proposing a system that will enable the tutor to select a template or create their own when there is no suitable one in the library. We accept that our library of templates and exemplar profiles will be restricted, but we do not feel this is an argument for not having templates. Tutors need access to a simple system that encapsulates best practice.

In our model we accept the fact that learners will have their personal profiles and that:

... resources are matched at delivery time with functional requirements of learners or users that are relevant to that immediate context.

*Corresponding author. Accessibility Research Centre, School of Computing, University of Teesside, Middlesbrough TS1 3BA, UK. Email: s.j.green@tees.ac.uk 
However, the point about profiles being anonymous is more than the fact that they are geared to a learner and context, in that there is no need for an individual to identify his or her disabilities or dysfunctions. From this we might propose that needs, preferences and contexts can be anticipated in the abstract or general sense. There is no suggestion, however, that this is anything other than a pragmatic position. Clearly any number of contexts, needs or preferences might be encountered that were not actually anticipated. However, as with allowing personalised web interfaces based on style-sheets, it is usually best if a user starts by modifying an existing style-sheet rather than specifying all elements from scratch.

The second point about the IMS AccessForAll approach is 'that the components that comprise a delivered resource can be assembled from distributed sources'.

Ultimately we would agree that our TASS would use a global federated search to assemble its components, and as such would be an instance of an AccessForAll service. However, the initial idea is to trial the service using only localised searches. (As it happens, Teesside subscribes to the LRC3 catalogue from University of NSW, Sydney Australia, which is federated with MERLOT and other international catalogue services, giving direct access to a very large number of learning objects.) Our view is that even a simple, localised service would need more intelligence than we can initially anticipate. For example, a tutor determines that they wish to employ a learning pattern based on an initial description of a concept, an animated simulation and a multiple choice test. It is not obvious from this description, without knowing more details of the subject matter and context of the learning activity, whether an audible explanation of the simulation would make any sense to a person who had expressed a need for a non-visual presentation. The probability in this instance would be that an alternative learning pattern (rather than a substitution for the simulation component) would be needed.

Here it might be appropriate to take some of Andy Heath's comments in more detail:

The existence of a generic anonymous set of preferences is not the same as the preferences of an individual learner. It is like a 'type' statement for a variable as can occur in a computer program. It is not an example value.

We suggest that TASS would respond directly to 'channels' rather than full profiles. A number of channels could form a profile and that might be regarded as a 'type' but we are providing instantiations (real values) for these types, from which a user can select. The implication that potential users will have little or no input is not the case. Whether the user would have enough input is open to debate. In our experience, users can be given too much choice as well as too little, especially if that choice is not fully informed.

It is not distinct from the notion of a Universally Accessible Resource, which the paper rightly points out is a flawed notion because it is not achievable ... Design patterns and guidelines are useful but more work is needed to explore how they may be used in practice without being misused.

Learning patterns allow us to separate the various parts of a learning object based on a functional hierarchy ranging from the pedagogical design approach to media 
component. We can then perform standard transformations, augmentations and substitutions that will have a good chance of working. This is not aiming at a universal resource at all, but rather utilising a set of known patterns that are useful in a particular context.

In an AccessForAll world, where components of a learning object can be assembled at or close to run time .... the person that authored the adaptation may not be the person that had the design template in mind. It seems that the notion of design templates may require that all authors become Accessibility-Aware. This is not likely to happen.

On the contrary, we take the view that all staff should be accessibility-aware. It is a legal requirement, after all, that resources should be accessible. That is not to say that all staff need the skills to produce accessible material; our approach provides a mechanism for producing materials that are adaptable (although not universally accessible) without the need for specialist skills. However, there will always be specific needs that are not catered for by patterns and templates, and these must be addressed by skilled authors. We do not deny this. As we say in the paper, the production of truly accessible learning objects is liable to be a collaborative activity.

We agree with the argument that classroom teachers do use 'design templates' because they know what patterns will and will not work, but that sometimes 'we need to just break all the rules and do something different because it works'. No set of learning patterns, however exhaustive, will cater for every eventuality, but the fact that design templates are not always useful is not an argument for not using them at all. However, we accept the caution here and echo the words of the IMS AccessForAll working group that we do not expect to get it right first time (or even second, third or fourth time). We expect to learn from our experiences and refine our model.

In this analysis it seems to me that design templates in this context represent a half-way house between the world where we say to learners 'here is what I think you need to access this stuff' and a world where learners say 'here is what I need to access this stuff'.

We agree whole-heartedly with this analysis. However our approach is not particularly novel: it is normal that authors produce content in the form that they think will be useful. It is then refined through feedback from its users. We also expect to evaluate our TASS as well as the IMS AccessForAll and Dublin Core 'Adaptability' standards as part of the process.

Finally, the questions posed:

1. What is the value of talking about groups of learners?

We wish to find commonality in needs and preferences where possible. The ability to deal with groups allows us to work at a higher level of abstraction and should make anticipation of individual needs more effective. Learner profiling with people with disabilities in FE has proven to be an effective approach in the past. However, we also accept that ultimately any service has to be responsive to individual needs and preferences.

2. How can we ensure Design Templates are not misused? 
We can never prevent misuse. However, with proven guidelines, staff development, specialist support and increased awareness we would expect tutors to become more familiar with the potential of such templates, and therefore better able to employ them.

3. How can Design Templates cater for repressed demand - where a user may only select from what is available and not know to say 'I want this'?

A Design Template can only cater for the type of demand that the tutor has directly anticipated. That may or may not be matched by an appropriate transformation, augmentation or substitution. If this is not catered for directly because the alternative component cannot be provided, then our service (the TASS) would defer to a wider global service (IMS AccessForAll) if and when that service became available. Additionally, the mismatch would provide information that would allow interested parties to provide the alternate component at a later date.

SENDA requires an Institution to be 'anticipatory' in meeting the needs of disabled students. Needs are often met as a result of identified barriers, resulting in 'just in time' developments. Even where a less than ideal compromise has to be made, the need can now be 'anticipated' for future learning experiences.

4. How can Design Templates be used across systems and organisations in ways that remain responsive to real individual requirements?

Patterns and templates are derived from successful use of similar resources. Thus they meet a known requirement. Adaptation to individual needs and preferences will require more work (as suggested earlier) but we need to start somewhere. Adaptations will be refined through evaluation and feedback, with the new knowledge gained being fed back into the TASS design. Designers of systems need to be closer to the human experience than is often the case: in a sense we are suggesting adopting a 'human systems' rather than a 'computer science' or 'programming' approach to designing our TASS and adaptable learning objects.

However, we should also remember that adaptability is not the whole requirement; a learning object needs to meet the learning outcomes and maintain its pedagogical integrity. The Learning Technology Research Institute's work, on which we are building, was based on the development of learning objects that are pedagogically sound and have been thoroughly evaluated to prove their value to learners. That is part of the reason for adopting the patterns on which they are based. We are trying to enrich the resources by making them both pedagogically sound and adaptable.

To conclude, the debate is not over. We need a better understanding of the use of learning patterns and learner profiles over a much wider range of contexts than we have so far been able to explore. Currently we are working on the design of the proposed tutor tools and TASS service as well as planning for the collection of a set of exemplar profiles of needs and preferences. This research represents a synergy of 
the work on learning patterns and generative learning objects at the Learning Technology Research Institute, London Metropolitan University and on accessible web services and learner profiles at the Accessibility Research Centre, University of Teesside. However, this collaboration, although productive, is still in its infancy. 